\title{
Production of SARS-CoV-2 virus-like particles in insect cells
}

Youjun $\mathrm{Mi}^{\mathrm{a}}$, Tao Xie ${ }^{\mathrm{a}}$, Bingdong Zhu ${ }^{\mathrm{a}^{*}}$, Jiying Tan ${ }^{\mathrm{b}}$, Xuefeng Lic, Yanping Luo ${ }^{\mathrm{b}}$, Fei $\mathrm{Li}^{\mathrm{a}}$, HongXia Niu ${ }^{\mathrm{a}}$, Jiangyuan Han ${ }^{\mathrm{a}}$, Wei Lv ${ }^{\mathrm{a}}$, Juan Wang ${ }^{\mathrm{a}}$

${ }^{a}$ Gansu Provincial Key Laboratory of Evidence Based Medicine and Clinical Translation and Lanzhou Center for Tuberculosis Research, School of Basic Medical Sciences, Lanzhou University ${ }^{\mathrm{b}}$ Department of Immunology, School of Basic Medicine, Lanzhou University

${ }^{\mathrm{c}}$ Department of Combined Western and Chinese Traditional Medicine, Lanzhou University Running title: SARS-CoV-2 virus-like particles

*Address corresponding to Bingdong Zhu, zhubingd@163.com

\begin{abstract}
Coronavirus disease (COVID-19) causes a serious threat to human health. To production of SARS-COV-2 virus-like particles (VLPs) in insect cells for vaccine development and scientific research. The E, M and S genes were cloned into multiple cloning sites of the new triple expression plasmid with one p10 promoter, two $\mathrm{pPH}$ promoters and three multiple cloning sites. The plasmid was transformed into DH10 $\mathrm{Bac}^{\mathrm{TM}}$ Escherichia coli competent cells to obtain recombinant bacmid. Then the recombinant bacmid was transfected in ExpiSf9 ${ }^{\mathrm{TM}}$ insect cells to generate recombinant baculovirus. After ExpiSf9 ${ }^{\mathrm{TM}}$ infected with the recombinant baculovirus, the E, M, and S protein co-expressed in insect cells. Finally, SARS-CoV-2 VLPs were self-assembled in insect cells after infection. The morphology and the size of SARS-CoV-2 VLPs are similar to the native virions.
\end{abstract}

Key words: SARS-CoV-2, virus-like particles, insect cells 
Coronavirus disease (COVID-19), caused by the severe acute respiratory syndrome coronavirus-2 (SARS-CoV-2), went on to ravage the world and caused the biggest pandemic 21 st century. Compared with severe acute respiratory syndrome coronavirus (SARS-CoV) and Middle East respiratory coronavirus (MERS-CoV), severe acute respiratory syndrome coronavirus 2 (SARS-CoV-2) spreads faster with an infection index of about $2.6[1,2]$. On October 15, 2020, more than 38.69 million people worldwide have been infected with the SARS-CoV-2, of which more than 1090,000 have died [3]. At present, there are no specific drugs for the coronavirus disease 2019 (COVID-19), many efforts have focused on neutralizing antibody and vaccine development [4]. Vaccines are the most economical and effective means to prevent and control infectious diseases [5]. There is an urgent need to develop SARS-CoV-2 vaccines to prevent and control the spread of the virus.

While many efforts are being made to design and develop vaccines for SARS-CoV-2. The World Health Organization estimates that there are about 133 vaccines under development [6]. Coronavirus vaccines generally fall into one of the following types: inactive or live-attenuated viruses, protein-based, virus-like particles (VLPs), viral vectors, and nucleic acid vaccines[7]. Inactivated viruses or live attenuated viruses require a large number of viruses to be cultured under biosafety level 3 (BSL3) conditions, and extensive safety testing is required, but the process is expensive, laborious and has a high safety risk. Protein-based subunit vaccines have poor immunogenicity due to incorrect folding of the target protein or poor display to the immune system, and require the addition of an adjuvant to induce a strong immune response. Nucleic acid vaccines cannot enter cells efficiently, need to be electroporated after injection, and mRNA is not very stable, multiple inoculations are required [8]. As a specific type of subunit vaccine, VLPs can mimic the natural morphology and structure of viruses, and have the characteristics of not containing viral genome nucleic acid, inability to replicate, and repeated vaccination of individuals. Because of VLPs strong immunogenicity, ability to elicit protective neutralizing antibodies and reliable safety, VLPs are strong candidates for vaccines design. At present, several vaccines based on VLPs are commercially available, these include human papillomavirus (HPV) 
vaccine and hepatitis B vaccine (HBV) [9]..

SARS-CoV, SARS-CoV-2 and MERS-CoV are all belong to the group of Betacoronaviruses $(\beta \mathrm{CoVs})$ and have similar structures [10]. According to previous studies, the composition of SARS-CoV VLPs [6] and MERS-CoV VLPs [8] required the E, M, and S proteins co-expression in the cells. Similar to other $\beta \mathrm{CoVs}$, The $3^{\prime}$ end of the SARS-CoV-2 genome encodes 4 main structural proteins, including the spike (S) protein, the envelope $(\mathrm{E})$ protein, the membrane $(\mathrm{M})$ protein and the nucleocapsid $(\mathrm{N})$ protein [11]. We speculate that SARS-CoV-2 VLP also consists of the E, M and the S protein. In this study, a triple expression plasmid that co-expression of the E, M, S proteins was constructed. The Bac to Bac baculovirus insect expression system was used to achieve the co-expression of the E, M, and S proteins in ExpiSf9 ${ }^{\mathrm{TM}}$ cells. Eventually, the E, M, S proteins self-assemble to form VLPs in the cell.

\section{MATERIALS AND METHODS}

\section{Cell lines}

ExpiSf9 ${ }^{\mathrm{TM}}$ cells were presented by Mr. Ru Yi from the State Key Laboratory of Lanzhou Institute of Veterinary Medicine, Chinese Academy of Agricultural Sciences. Expi ${ }^{\mathrm{TM}} \mathrm{Sf9}$ cells were maintained as suspension cultures in flasks with serum-free ExpiSf ${ }^{\mathrm{TM}} \mathrm{CD}$ Medium (Gibco, USA) at $27^{\circ} \mathrm{C}$ with stirring at a speed of $125 \mathrm{rpm}$. Cell density was determined by microscopically counting the number of cells and cell viability was judged by trypan blue dye exclusion.

\section{Construction of the EMS triple expression plasmid}

The dual expression plasmid pFastBacDual (Invitrogen, USA) was purchased from Thermo Fisher Scientific China Co., Ltd.; In order to obtain a single recombinant baculovirus that co-expressed the $\mathrm{M}, \mathrm{E}$ and $\mathrm{S}$ proteins, a new triple expression vector was generated. In brief, a new SV40 poly A tail, a new pPH promoter and a NdeI restriction site were inserted into the EcoRI and HindIII restriction sites after pPH promoter of $\mathrm{pFastBacDual}$. The new triple expression vector has one $\mathrm{p} 10$ promoter and two $\mathrm{pPH}$ promoters. For co-expression of the E, M, and S proteins, the codon optimized 
E, M, S genes of SARS-CoV-2 (GenBank accession No. MN908947.3) cloned into the triple expression vector. The E gene cloned into the double KpnI and XhoI restriction sites under the control of the p10 promoter, the $\mathrm{M}$ gene inserted into the BamHI and EcoRI restriction sites under the control of the pPH promoter, and the $\mathrm{S}$ genes cloned into the double NdeI and HindIII restriction sites under the control of other pPH promoter. Finally, the triple expression plasmid named EMS was generated and verified by DNA sequencing (BGI, China).

\section{Generation of recombinant baculovirus}

Recombinant baculovirus were generated by using a Bac-to-Bac expression system (Invitrogen, USA) according to the manufacturer's instructions. Briefly, the EMS plasmid was transformed into Top10 competent (Transgen, China). After re-extracting the EMS plasmid, it was transformed into DH10 $\mathrm{Bac}^{\mathrm{TM}}$ E. coli competent (Invitrogen, USA). White colonies were screened in LB media containing the antibiotics gentamicin $(7 \mu \mathrm{g} / \mathrm{ml})$, tetracycline $(10 \mu \mathrm{g} / \mathrm{ml})$, kanamycin $(50 \mu \mathrm{g} / \mathrm{ml})$ and X-Gal (5-bromo-4chloro-3-indolyl- $\beta$-D-galactopyranoside) and IPTG (isopropyl- $\beta$-Dthiogalactopyranoside). After twice cycles of white colony screening, recombinant Bacmid DNA were isolated and transfected into ExpiSf9 ${ }^{\mathrm{TM}}$ cells. Transfected ExpiSf $9^{\mathrm{TM}}$ cells cultured at $27^{\circ} \mathrm{C}$ and $125 \mathrm{rpm}$, until the cytopathic rate reached $30 \%$, the cell supernatant was collected to obtain the recombinant baculovirus. Using PCR to verify the genes of interest in the recombinant bacmid. The primers for PCR are shown in Table 1.

Table 1 Analyze recombinant bacmid DNA by PCR

\begin{tabular}{|c|c|c|c|}
\hline Genes & Size of PCR Product & primers & Sequence $\left(5^{\prime}-3^{\prime}\right)$ \\
\hline \multirow{2}{*}{$\mathrm{E}$} & \multirow{2}{*}{$228 b p$} & $\mathrm{EF}$ & ATGTACTCATTCGTTTCGGA \\
\hline & & ER & TTAGACCAGAAGATCAGGAACTC \\
\hline \multirow{2}{*}{ M } & \multirow{2}{*}{$669 \mathrm{bp}$} & MF & ATGGCAGATTCCAACGGTA \\
\hline & & MR & TTACTGTACAAGCAAAGCAATATT \\
\hline \multirow{2}{*}{$\mathrm{S}$} & \multirow{2}{*}{$3819 b p$} & SF & ATGTTTGTTTTTCTTGTTTTATTG \\
\hline & & SR & TTATGTGTAATGTAATTTGACTCCTTT \\
\hline \multirow{2}{*}{$S^{\prime}$} & \multirow{2}{*}{ 1486bp } & $S^{\prime} F$ & AAACACGCTTGTTAAACAAC \\
\hline & & M13R & CAGGAAACAGCTATGAC \\
\hline
\end{tabular}




\section{Preparation of EMS VLPs}

Incubating ExpiSf9 ${ }^{\mathrm{TM}}$ cells for 18 hours, add ExpiSf ${ }^{\mathrm{TM}}$ Enhancer (Invitrogen, USA) to the cell culture. 24 hours after ExpiSf ${ }^{\text {TM }}$ Enhancer addition, infect cells with baculovirus in a volume of 50:1. Cells were collected on day 4 by centrifugation at $3000 \mathrm{~g}$ for 5 minutes, freeze-thaw repeatedly for 3 times, and discard the pellet after centrifugation at $8000 \mathrm{~g}$ for 30 minutes. The supernatant was ultra-centrifuged at $100000 \mathrm{~g}$ for 1 hour at $4{ }^{\circ} \mathrm{C}$, and the pellets were resuspended in phosphate-buffered saline (PBS) at $4^{\circ} \mathrm{C}$ overnight. EMS VLPs were through a 30\%-40\%-50\% discontinuous sucrose gradient at $100,000 \mathrm{~g}$ for 2 hours at $4^{\circ} \mathrm{C}$. The white bands between $30-40 \%$ were collected and diluted with PBS and pelleted at $100000 \mathrm{~g}$ for 1 hour at $4^{\circ} \mathrm{C}$. The VLPs was collected and resuspended in PBS overnight at $4{ }^{\circ} \mathrm{C}$. EMS VLPs were store $-80^{\circ} \mathrm{C}$ for the following analysis.

\section{Western Blot Analysis}

The VLPs were characterized by Western blot analysis and electric microscopic observation. For Western blot analysis, VLPs sample were subjected to SDS-PAGE using a $10 \%$ gel, followed by transfer to poly vinylidene difluoride (PVDF) membranes. The PVDF membranes were then blocked with TBST (10 mM Tris- $\mathrm{HCl}, 150 \mathrm{mM} \mathrm{NaCl}$, $0.5 \%$ Tween 20 ) containing $5 \%$ skim-milk powder. The S protein of VLP was detected by Western blotting, using an anti-SARS-CoV-2 S polyclonal rabbit antibody (Sino Biological, China). The expression of the $\mathrm{E}$ and the $\mathrm{M}$ protein were also verified by polyclonal antibodies (data not shown). Alkaline phosphatase-conjugated goat-antirabbit IgG (immunoway, China) were used as the secondary antibodies to label the protein bands.

\section{Electron microscopy}

For transmission electron microscopy. 5ul VLPs samples were applied onto a carboncoated film. 2 minutes later, the samples was removed with filter paper. Then, $8 \mu 1$ of $1 \%$ phosphotungstic acid was applied onto the grid, and the samples were stained for 
60s. The staining solution was removed with filter paper, and the grid was dried for 30 min at room temperature. After being stained, the sample was observed using a FEI Talos F200C transmission electron microscope (FEI, Czech Republic) at $200 \mathrm{kV}$ and 100-200 k-fold magnification.

\section{Results}

\section{Construction of EMS triple expression plasmid}

To co-expression the SARS-CoV-2 E, M, S proteins in insect cells, a new triple expression vector was constructed. The triple expression vector contains one pP10 promoter, two pPH promoters and three multiple cloning sites (Fig1 b). Then the SARS$\mathrm{CoV}-2 \mathrm{E}, \mathrm{M}$ and $\mathrm{S}$ genes were cloned into multiple cloning sites respectively to generate EMS plamsid (Fig1 c).
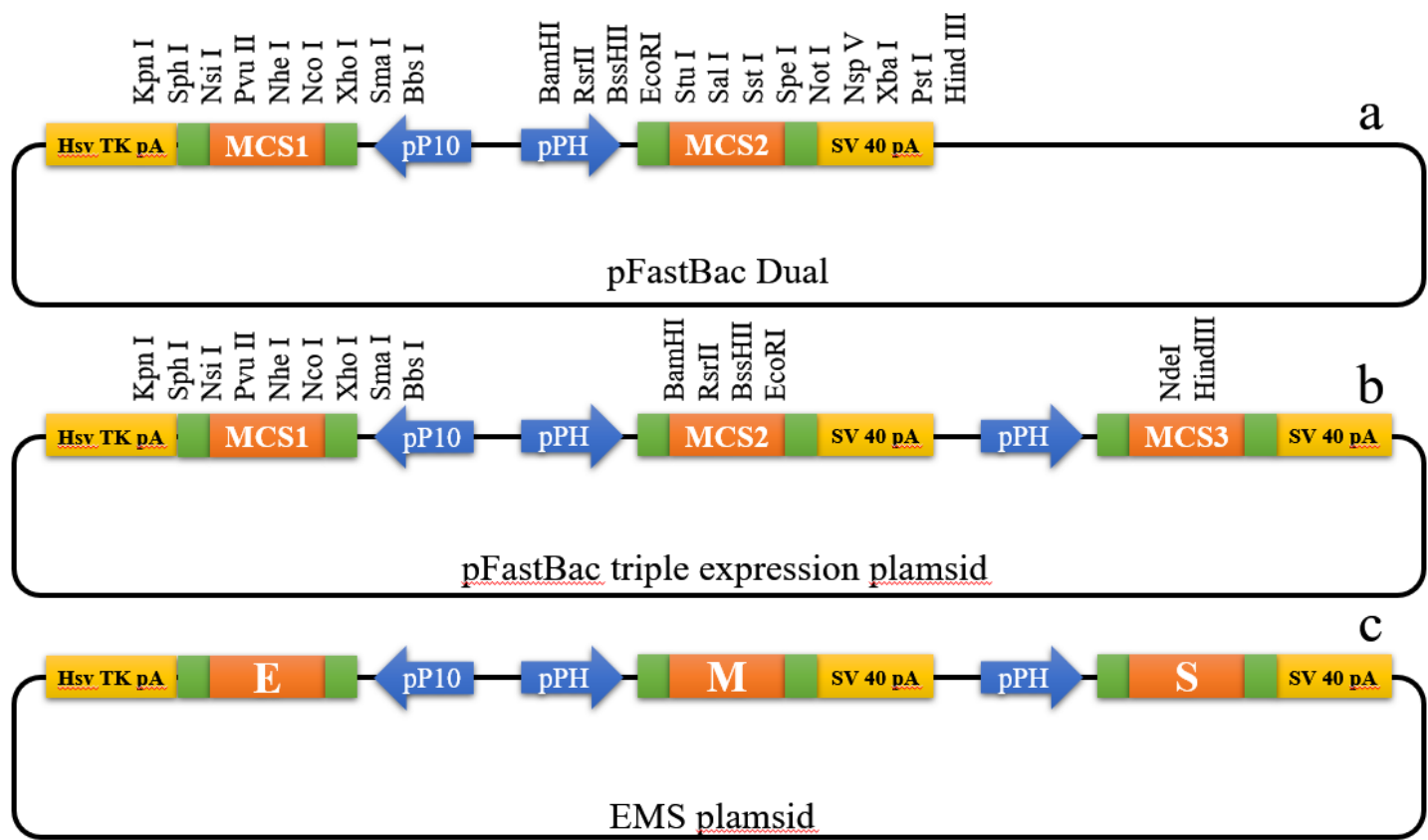

Fig 1 Construction of EMS plasmid

a pFastBac Dual b pFastBac triple expression plasmid c EMS plasmid

\section{Generation of recombinant bacmid DNA}

The EMS recombinant plasmid was transformed into $\mathrm{DH} 10 \mathrm{Bac}^{\mathrm{TM}} E$. coli competent Cells. Blue and white colonies were visible on the LB screening plate after 48 hours (Fig. 2a). Pick white colonies for the second screening to obtain positive colonies which containing the recombinant bacmid (Fig. 2b). To verify the E, M, and $\mathrm{S}$ genes in the 
recombinant bacmid by using PCR analysis. The target bands can be obtained at the corresponding positions (Fig. 3)
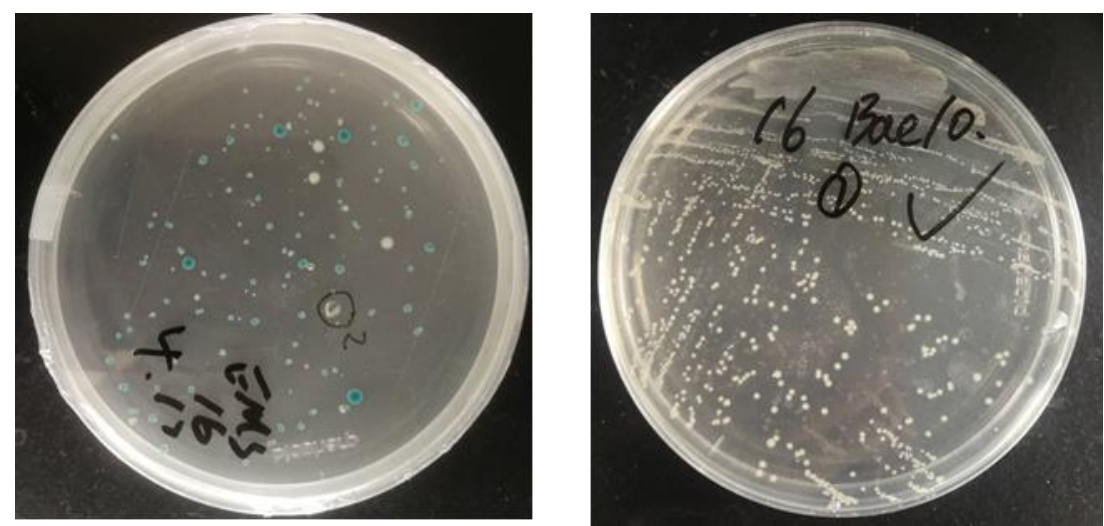

Fig 2 Transformation and secondary screening

a The EMS vector was transformed into DH10 $\mathrm{Bac}^{\mathrm{TM}}$ competent cells

b Secondary screening after transformation

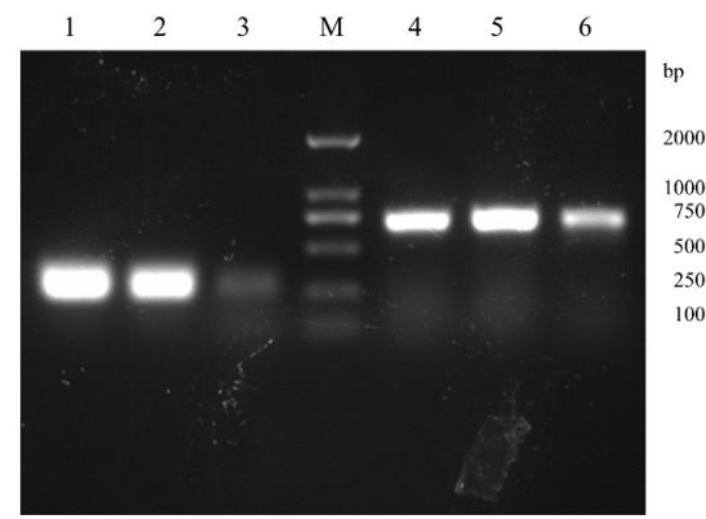

a

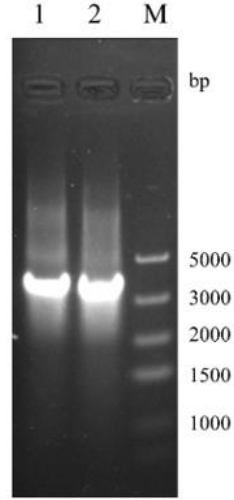

b

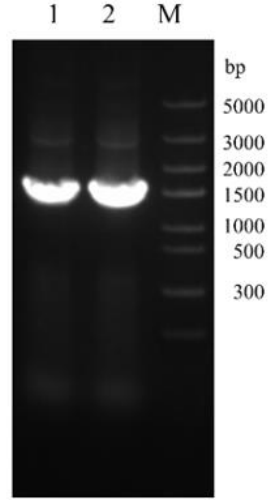

C

Fig 3 Verify the phenotype

a 1,2,3 Identification of the E gene by PCR;4,5,6 Identification of the M gene by PCR b 1,2 Identification of the S gene by PCR c 1,2 Identification of S' DNA fragment by PCR

\section{Recombinant baculovirus production}

For producing of recombinant baculovirus, isolating bacmid DNA to transfect ExpiSf9тм insect cells. After the baculovirus is added to the cells, observation the signs of infection. The swollen cells with enlarged nuclei indicate that the cell is infected by baculovirus (Fig. 4). After trypan blue staining, the cell death rate was increased. Collecting the recombinant baculovirus from the cell culture medium when the cells 
appear characteristics typical of late to very late infection.

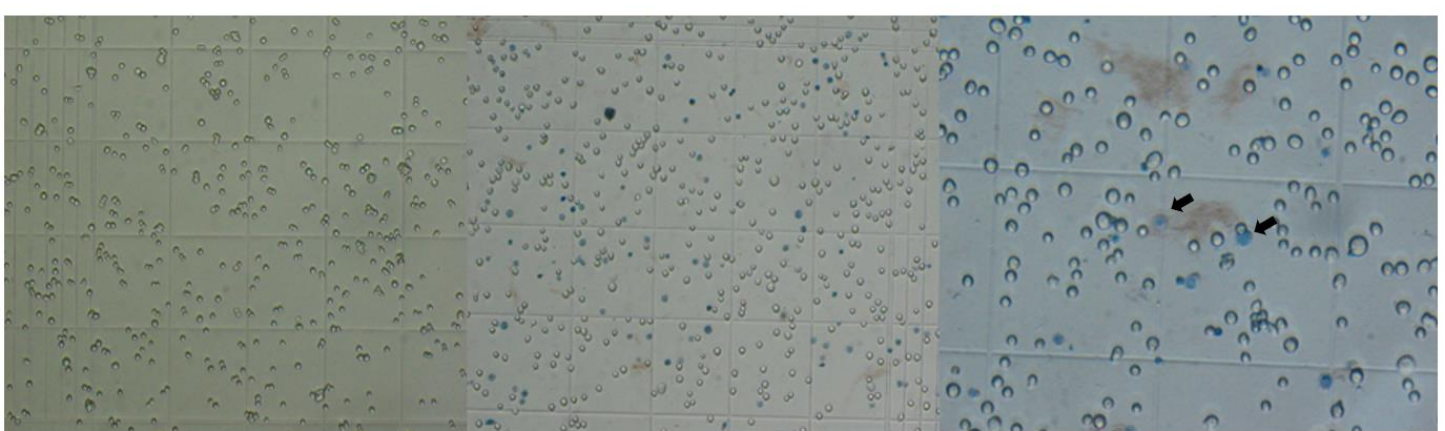

a

b

c

Fig 4 Cytopathic signs observed in ExpiSf $9^{\mathrm{TM}}$ cells

a $\quad$ ExpiSf9 $9^{\mathrm{TM}}$ cells $(\times 10) \quad$ b Transfected ExpiSf9 $9^{\mathrm{TM}}$ cells $(\times 10)$

c Transfected ExpiSf9 ${ }^{\mathrm{TM}}$ cells $(\times 40)$

\section{Production and characterization of SARS-CoV-2 VLPs}

To product SARS-CoV-2 VLPs in insect cells, Sf9 cells were infected with recombinant baculovirus. 96 hours after infection, the cells were harvested and SARS-CoV-2 VLPs were purified by sucrose gradient centrifugation. To confirm the $\mathrm{S}$ protein was incorporated within the VLPs, purified VLPs were analyzed by Western blot using SARS-CoV-2 S protein polyclonal antibody. The result showed that VLPs contained SARS-CoV-2 S proteins (Fig 5). The morphology of SARS-CoV-2 VLPs was investigated by electron microscopy. The SARS-CoV-2 VLPs exhibited spheriform structures, and the diameters of VLPs were approximately $100 \mathrm{~nm}$ (Fig 6). These results indicated that SARS-CoV-2 VLPs autonomously assemble in insect cells infected with recombinant baculovirus, and are structurally similar to the native virions[12]. 


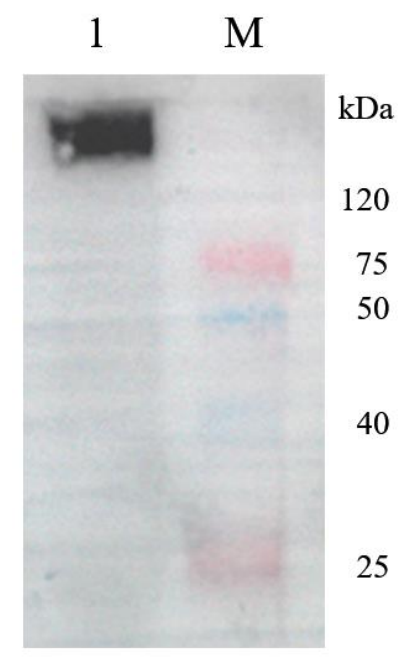

Fig.5 Western-blotting identification of SARS-CoV-2 S protein expressed on VLPs 1 identification of SARS-CoV-2 S protein with S antibody M Protein maker

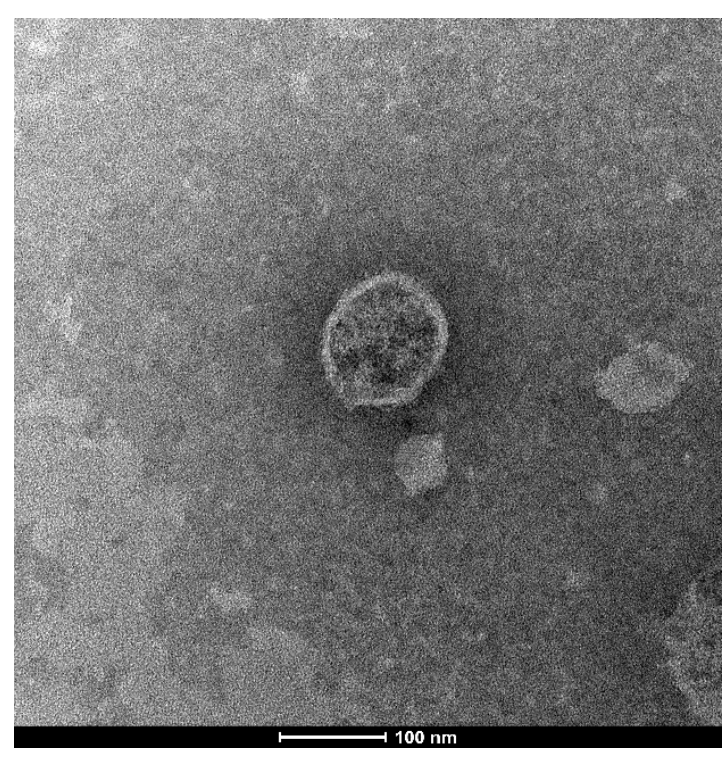

Fig 6 VLP observed under electron microscope

\section{CONCLUSION}

Although some countries have successfully controlled the COVID-19 epidemic, on the face of ongoing death and destruction caused by SARS-CoV-2 virus, the urgent need for developing vaccines to against the spread of the virus. At present, SARS-CoV-2 related traditional vaccines and new generation vaccines are under development, but there is no effective vaccine approved for use. Because of their unique advantages, VLPs have been use as vaccine platform. Baculovirus system is especially suitable for virus-like particle production, since this system allows more than one gene to be co- 
expressed in a cell.

SARS-CoV-2, SARS-Cov, and MERS-CoV are all belong to $\beta-\mathrm{CoV}$, have similar virus structures which consist of 4 structural proteins N, E, M, S[11]. Eduardo Mortola reported that the M, E and S proteins self-assembled to form SARS-CoV VLPs when co-transfection of the E, M, and S baculovirus in Sf9 insect cells[13]. Xinya $\mathrm{Lu}$ confirmed that SARS-Cov VLPs are immunogenic and can cause strong SARS-Cov VLPs specific humoral and cellular immune responses in mice [14]. Similarly, the coinfection of MERS CoV E, M and S recombinant baculoviruses in insect cells produces VLPs with similar morphological signature to the native virions. MERS-CoV VLPs are immunogenic, able eliciting robust levels of specific humoral and cell-mediated immunity in rhesus monkeys. Rhesus monkeys vaccinated MERS-CoV VLPs with alum adjuvant induced high titer virus neutralizing antibodies and triggered $\mathrm{T}$ helper 1 cells (Th1) mediated immunity[15].

In this study, a new triple expression vector was constructed, then the E, M, and S gene were cloned into the triple expression vector to generate EMS plasmid. The EMS vector was transformed into DH10 $\mathrm{Bac}^{\mathrm{TM}}$ competent cells, and the recombinant bacmid was obtained after twice screening. Then the recombinant bacmid was transfected in ExpiSf9 ${ }^{\mathrm{TM}}$ insect cells to obtain recombinant baculovirus. After ExpiSf9 ${ }^{\mathrm{TM}}$ infected with the recombinant baculovirus, the $\mathrm{E}, \mathrm{M}$, and $\mathrm{S}$ protein co-expressed in cells to form VLPs by self-assembly. To our knowledge, a successful construction of SARS-CoV-2 VLPs via insect expression system has not yet been reported. In the future, humoral and cellular immunogenicity of SARS-CoV-2 VLPs in animal models will be further evaluated. In addition, VLPs can also be used to study the pathogenesis of COVID-19.

\section{ACKNOWLEDGEMENTS}

This study was Supported by the Fundamental Research Funds for the Central Universities (lzujbky-2020-sp06 and lzujbky-2017-22), supported by the Key research and development program of Gansu Province in 2020 (20YF8FA072) and by Lanzhou science and technology planning project(2020-XG-33). 


\section{REFERENCES}

1. Han, Q., et al., Recent insights into 2019-nCoV: a brief but comprehensive review. J Infect, 2020.

2. Han, Q., et al., Recent insights into 2019-nCoV: a brief but comprehensive review. Journal of Infection, 2020.

3. Organization., W.H. Global novel Coronavirus(2019-nCoV) Situation Report. 2020; Available from: https://www.who.int/emergencies/diseases/novel-coronavirus2019/situation-reports.

4. Lu, H., Drug treatment options for the 2019-new coronavirus (2019-nCoV). Biosci. Trends, 2020. 14(1): p. 69-71.

5. Rémy, $\mathrm{V}$., et al., The economic value of vaccination: why prevention is wealth. J. Mark. Access Health Policy, 2015. 3.

6. WHO, DRAFT Landscape of COVID-19 Candidate Vaccines. https://www.who.int/whodocuments-detail/draft-landscape-of-covid-19-candidate-vaccines, 2020.

7. Liu, C., et al., Research and Development on Therapeutic Agents and Vaccines for COVID19 and Related Human Coronavirus Diseases. ACS Cent. Sci., 2020. 6(3): p. 315-331.

8. van Riel, D. and E. de Wit, Next-generation vaccine platforms for COVID-19. Nat. Mater., 2020. 19(8): p. 810-812.

9. Chung, Y.H., H. Cai, and N.F. Steinmetz, Viral nanoparticles for drug delivery, imaging, immunotherapy, and theranostic applications. Adv. Drug Delivery Rev., 2020. S0169409X(20): p. 30070-30073.

10. Gralinski, L.E. and V.D. Menachery, Return of the Coronavirus: 2019-nCoV. Viruses, 2020. 12: p. 2.

11. Chan, J.F.-W., et al., Genomic characterization of the 2019 novel human-pathogenic coronavirus isolated from a patient with atypical pneumonia after visiting Wuhan. Emerging Microbes Infect., 2020. 9(1): p. 221-236.

12. China, N.P.M.R.B.O., Electron micrograph of Wuhan strain 01-02 of novel coronavirus. . 2020: p. Available from: http://www.nmdc.cn/nCoV.

13. Mortola, E. and P. Roy, Efficient assembly and release of SARS coronavirus-like particles by a heterologous expression system. FEBS Lett., 2004. 576(1-2): p. 174-178.

14. Lu, X., et al., Immune responses against severe acute respiratory syndrome coronavirus induced by virus-like particles in mice. Immunology, 2007. 122(4): p. 496-502.

15. Wang, C., et al., MERS-CoV virus-like particles produced in insect cells induce specific humoural and cellular imminity in rhesus macaques. Oncotarget, 2017. 8(8): p. 1268612694. 